\section{Perdas dentárias e fatores sociais, demográficos e de serviços associados em adultos brasileiros: uma análise dos dados do Estudo Epidemiológico Nacional (Projeto SB Brasil 2002-2003)}

\author{
Tooth loss and associated socioeconomic, \\ demographic, and dental-care factors in \\ Brazilian adults: an analysis of the Brazilian \\ Oral Health Survey, 2002-2003
}

Paulo Roberto Barbato ${ }^{1}$ Helen Cristhiane Muller Nagano 1 Fabiane Nunes Zanchet 1 Antonio Fernando Boing 1,2 Marco Aurélio Peres 1

\section{Introdução}

The aim of this study was to estimate the prevalence of tooth loss among Brazilian adults aged 35 to 44 years. The study also tested the association between tooth loss and demographic, socioeconomic, and dental-care utilization variables. Data were analyzed from 13,431 individuals submitted to dental examination and interviewed in the National Oral Health Survey in 2002-2003. The number of lost teeth ( $\leq 12$ and $>12)$ was the outcome. Exploratory variables included geographic area, gender, skin color, age, per capita income, schooling, time since last dental appointment, and type of dental service used. Crude and adjusted prevalence ratios were estimated using a Poisson regression model. The prevalence of edentulism was 9\%, while median tooth loss was 11. Tooth loss was strongly associated with living in rural areas, female gender, poor socioeconomic status, low schooling, and older age. Individuals who attended public dental care services and those with more time elapsed since their last dental appointment showed a higher prevalence of tooth loss than their counterparts after controlling for demographic and socioeconomic factors.

Tooth Loss; Oral Health; Health Inequalities; Adult
O edentulismo - perda total dos dentes - é um dos piores agravos à saúde bucal. A alta proporção de brasileiros com perda total de dentes, a possibilidade de controle desse agravo e os danosos impactos na vida das pessoas afetadas desafiam a saúde pública a minimizar esse problema. As perdas dentárias constituem-se em uma marca da desigualdade social, diminuem a capacidade mastigatória 1 , dificultando e limitando o consumo de diversos alimentos, afetam a fonação e causam danos estéticos que podem originar alterações psicológicas 2 . Esse conjunto de repercussões no cotidiano das pessoas contribui para a redução da qualidade de vida das mesmas 3 .

A cárie é a principal causa de perdas dentárias 4 .Os traumatismos dentários e as doenças periodontais também contribuem para estas perdas, porém em menor grau. Além destes agravos, a perda dentária é decorrente de atitudes dos profissionais da Odontologia 5 e da população, da acessibilidade e utilização de serviços odontológicos, da modalidade de financiamento do sistema de saúde e da forma de prestação de cuidados odontológicos 6,7. Motivos de ordem econômica são comuns como causa primária ou correlata de extrações dentárias 8 .

Historicamente, no Brasil, à população adulta têm sido destinados os serviços de urgências odontológicas, em sua grande maioria ocasiona- 
das por dor, o que via de regra resulta em perda dentária. Conseqüência direta dessa exclusão sistemática dos serviços, as extrações dentárias são desfechos que, na maioria das vezes, poderiam ser evitados 9 .

Proposto por Klein \& Palmer 10, em 1937, o índice CPO-D é um dos mais utilizados em saúde bucal. Esse índice é a expressão do número de dentes permanentes atacados por cárie através da soma do número de dentes permanentes cariados (componente "C" do índice), somados ao número de dentes "obturados" (componente "O") e ao número de dentes perdidos (componente "P”). Dados do levantamento epidemiológico nacional de saúde bucal realizado no Brasil em 1986 apontaram que na faixa etária dos 35 aos 44 anos o índice CPO-D médio foi de 22,5, com o componente "P" representando 14,96 dentes perdidos em média $\left(66,48 \%\right.$ do CPO-D) ${ }^{11}$. Em 2003, a média do índice CPO-D nesta mesma faixa etária era 20,13 , sendo o componente "P" responsável por 13,23 dentes em média $(65,72 \%$ do CPO-D) 12 , indicando um perfil de morbidade muito semelhante ao verificado há cerca de vinte anos para esta faixa etária.

Apesar de serem um problema de saúde pública, as perdas dentárias vêm sendo pouco investigadas no Brasil. Pesquisa realizada no MEDLINE, Biblioteca Brasileira de Odontologia (BBO) e LILACS utilizando como descritor de assunto "perda de dente", associado ao aspecto "epidemiologia", com os limites "humanos adultos”, no período de 1997 a 2006 (exceção à BBO - até 2005), nos idiomas "português", "espanhol" e "inglês", encontrou apenas uma publicação 13 . Pesquisa na base SciELO, utilizando os descritores "perda de dente", "perda dentária" e "tooth loss” identificou quatro publicações com a temática, porém apenas um estudo epidemiológico 14 . O pequeno número de publicações que tratam do tema e sua relevância como desfecho para a Saúde Pública ressaltam a necessidade de estudos sobre o mesmo.

No período entre 2003 e 2006, o Ministério da Saúde implementou em todo o território nacional mais de 400 Centros de Especialidades Odontológicas, unidades de atenção secundária em saúde bucal através das quais pretende-se oferecer, dentre outras possibilidades terapêuticas, a reabilitação e a manutenção da dentição 15 . O impacto destes serviços na preservação da dentição somente poderá ser avaliado no futuro.

O objetivo deste estudo foi o de estimar a prevalência de perdas dentárias em adultos brasileiros de 35 a 44 anos investigando associações deste agravo com condições demográficas, sócio-econômicas e com utilização de serviços odontológicos.

\section{Métodos}

Entre maio de 2002 e outubro de 2003 um total de 108.921 pessoas de diferentes faixas etárias, desde crianças com 18 a 36 meses de idade até idosos, foram entrevistadas e examinadas por pesquisadores do Projeto SB Brasil, em um levantamento epidemiológico de saúde bucal conduzido pelo Ministério da Saúde do Brasil 12.

A técnica de amostragem foi probabilística por conglomerados em três estágios, sendo selecionados, através de sorteio, 250 municípios de diferentes portes populacionais de todas as Unidades Federativas do Brasil.

Para cada Estado foram selecionados instrutores com experiência prévia em levantamentos epidemiológicos de saúde bucal. Estes instrutores promoveram o treinamento e a calibração de aproximadamente 900 cirurgiões-dentistas e 1.200 anotadores, adotando-se os critérios de diagnóstico da 4a edição do Oral Health Surveys: Basic Methods, da Organização Mundial da Saúde (OMS) 16 .

Os exames bucais dos adultos foram executados em ambientes internos dos domicílios, sob iluminação natural, usando sonda periodontal (CPI), espelho bucal plano e espátulas de madeira para melhorar a visualização do campo examinado. Aproximadamente 5\% dos exames foram executados em duplicata com o objetivo de mensurar a reprodutibilidade intra-observador.

Para os adultos foram observadas as características de cárie dentária de coroa e raiz, condições periodontais, necessidade de tratamento odontológico e uso e necessidade de prótese. Cada condição foi registrada em formulário padronizado para o levantamento. Através da aplicação de questionário especialmente elaborado para o estudo, também foram obtidos dados relativos à condição sócio-econômica do examinado, à utilização de serviços odontológicos e à sua autopercepção da saúde bucal. Maiores informações acerca da metodologia do estudo estão disponibilizadas no seu relatório final 11 .

Um total de 13.431 pessoas entre 35 e 44 anos foram examinadas pelo Projeto SB Brasil. Essa faixa etária é considerada padrão pela OMS para a avaliação das condições de saúde bucal em adultos 16 .

A variável dependente estudada foi a ocorrência de perdas dentárias, construída a partir da soma dos códigos 4 (perda dentária decorrente de cárie) e 5 (perda dentária decorrente de outras razões) do índice $\mathrm{CPO}-\mathrm{D}$ para o diagnóstico da coroa de cada um dos 32 espaços dentários examinados por indivíduo. Posteriormente, para a análise, esta variável foi dicotomizada em indivíduos com perdas de até 12 dentes e acima 
deste número. Este ponto de corte foi adotado de acordo com o referencial teórico do arco dentário reduzido, segundo o qual adultos devem manter um mínimo de vinte dentes funcionais, sem lacunas estéticas, de modo a permitir uma função mastigatória aceitável 17 .

Como variáveis independentes foram selecionadas: localização geográfica, gênero, cor da pele, idade, renda per capita, escolaridade, tempo decorrido desde a última consulta odontológica e tipo de serviço odontológico utilizado. As variáveis localização geográfica (rural ou urbana) e gênero (masculino e feminino) foram mantidas conforme disponibilizadas no banco de dados original. A variável cor da pele foi coletada segundo as categorias utilizadas nos recenseamentos promovidos pelo Instituto Brasileiro de Geografia e Estatística (IBGE), a saber: amarelos, brancos, pretos, pardos e indígenas. Para este estudo foram excluídos os indivíduos das categorias "amarelos", "indígenas" e aqueles sem cor de pele indicada, que totalizaram cerca de $4 \%$ da amostra. Pardos e pretos constituíram uma única categoria e brancos a outra. A idade, no banco original apresentada como uma variável numérica discreta - 35 a 44 anos - foi dicotomizada com base na mediana do intervalo ( 35 a 39 anos e 40 a 44 anos). A variável renda familiar, coletada como variável contínua, foi transformada em renda per capita dividindo-a pelo número de moradores do domicílio. Na seqüência, com base no salário mínimo vigente em de abril de 2002 (200,00 Reais ou 86,16 Dólares Americanos), foi dividida em duas categorias, utilizando como ponto de corte o valor de meio salário mínimo (indicador de renda insuficiente). A escolaridade, coletada como variável contínua (anos de estudos completados), foi dividida em três categorias com base no modelo de ensino vigente no país na época em que os indivíduos pesquisados freqüentavam a escola (mais de 8 anos, 5 a 8 anos e até 4 anos de estudo). A variável tempo decorrido desde a última consulta odontológica assumiu a distribuição ordinal com três categorias (até 1 ano, 1 a 2 anos e mais de 2 anos). A proporção de indivíduos que nunca se consultou com dentista atingiu apenas $2,8 \%$ da amostra, sendo excluída da análise. O tipo de serviço utilizado, categorizado originalmente como "nunca foi ao dentista”, "público", "liberal”, "convênio", "filantrópico" e "outros" foi agrupado como público e privado, este último agrupando liberal e convênio, tendo sido suprimidas as demais categorias em razão de sua pequena participação na amostra.

As variáveis foram agrupadas em blocos, de acordo com um modelo teórico hierárquico de determinação das perdas dentárias (Figura 1). Neste modelo, a localização geográfica de mo- radia dos indivíduos foi situada na posição mais distal em relação ao desfecho, por ter sido considerada como determinante para os demais agrupamentos. Admite-se que a localização no meio rural condiciona menores níveis de escolaridade e renda em comparação ao meio urbano. Da mesma forma os serviços de saúde são limitados, tanto em oferta quanto em relação às possibilidades de acesso. Condições sócio-econômicas, como renda e escolaridade influenciam o padrão e o tipo de utilização de serviços odontológicos. Gênero, idade e cor da pele influenciam a renda e a escolaridade, enquanto o gênero e idade determinam o padrão de utilização de serviços de saúde.

Inicialmente, comparou-se o número de perdas dentárias segundo as categorias de cada uma das variáveis independentes através do teste de Mann-Whitney, para variáveis dicotômicas, e Kruskal-Wallis quando havia mais de três categorias. A opção pelos testes não paramétricos resultou da observação da assimetria na distribuição das perdas dentárias.

Realizaram-se as análises univariadas das proporções de perda dentária para cada variável independente, apresentando as razões de prevalência com os respectivos intervalos de confiança de 95\% (IC95\%) e p valor.

Posteriormente, para estimar as razões de prevalência bruta e ajustada e seus respectivos IC95\% e p valor (obtido através do teste de Wald), foi realizada a regressão de Poisson com estimação de variância robusta. A opção pela regressão de Poisson deve-se ao fato de que em situações onde a prevalência do desfecho excede a $20 \%$ a utilização da regressão logística para a estimativa das razões de chances (odds ratio - OR) superestima a medida de efeito 18 .

A modelagem estatística seguiu o modelo teórico hierárquico de determinação das perdas dentárias. As variáveis do bloco 2, sócio-econômicas e demográficas, foram ajustadas entre si e pela variável mais distal do bloco 1 (região geográfica). Posteriormente, as variáveis relativas aos serviços odontológicos (bloco 3) foram ajustadas entre si e pelas variáveis superiores (blocos 2 e 1 ).

Todas as análises foram realizadas através do programa Stata 9.0 (Stata Corp., College Station, Estados Unidos) utilizando os comandos svy para análise de dados oriundos de amostras complexas. Este ajuste se torna necessário porque indivíduos dentro de um cluster possivelmente são mais similares entre si do que quando comparados com os de outros clusters. Quanto maior o número de indivíduos por cluster, maior será o efeito de desenho (deff), assim como quanto maior o coeficiente de correlação intraclasse, 


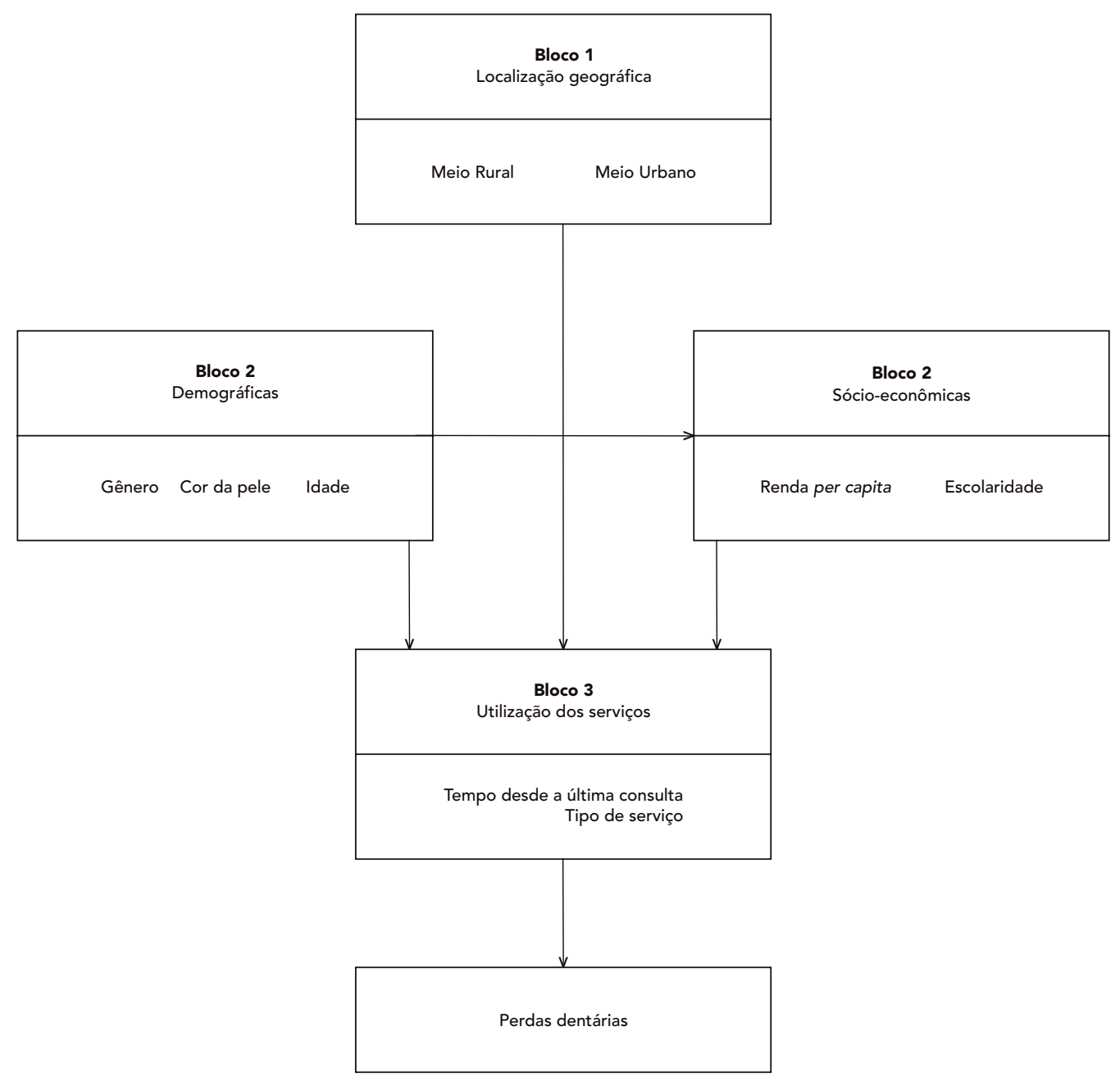

maior o deff. Estimativas que não levam em consideração a organização por cluster da amostra tendem à superestimação, assim é necessário proceder ao ajuste pelo efeito de amostragem 19 .

$\mathrm{O}$ projeto de pesquisa original (Projeto SB Brasil 2003) foi submetido à Comissão Nacional de Ética em Pesquisa (CONEP) e aprovado em 21 de julho de 2000, de acordo com o parecer no. $581 / 2000$.

\section{Resultados}

Foram analisados os dados referentes a 12.811 participantes do estudo residentes em 250 municípios brasileiros.

Na Figura 2 são apresentados os números de dentes perdidos distribuídos entre os participantes do estudo. Observa-se que a freqüência de distribuição das perdas dentárias é assimétrica. $\mathrm{O}$ maior número de participantes tem entre $4 \mathrm{e}$ 8 dentes perdidos, representando aproximadamente $24 \%$ da amostra e o menor número entre 16 e 20 dentes perdidos (aproximadamente 10\%). A prevalência de edentulismo (perda dos 32 dentes) nesta faixa etária atingiu 9\%, que correspon- 


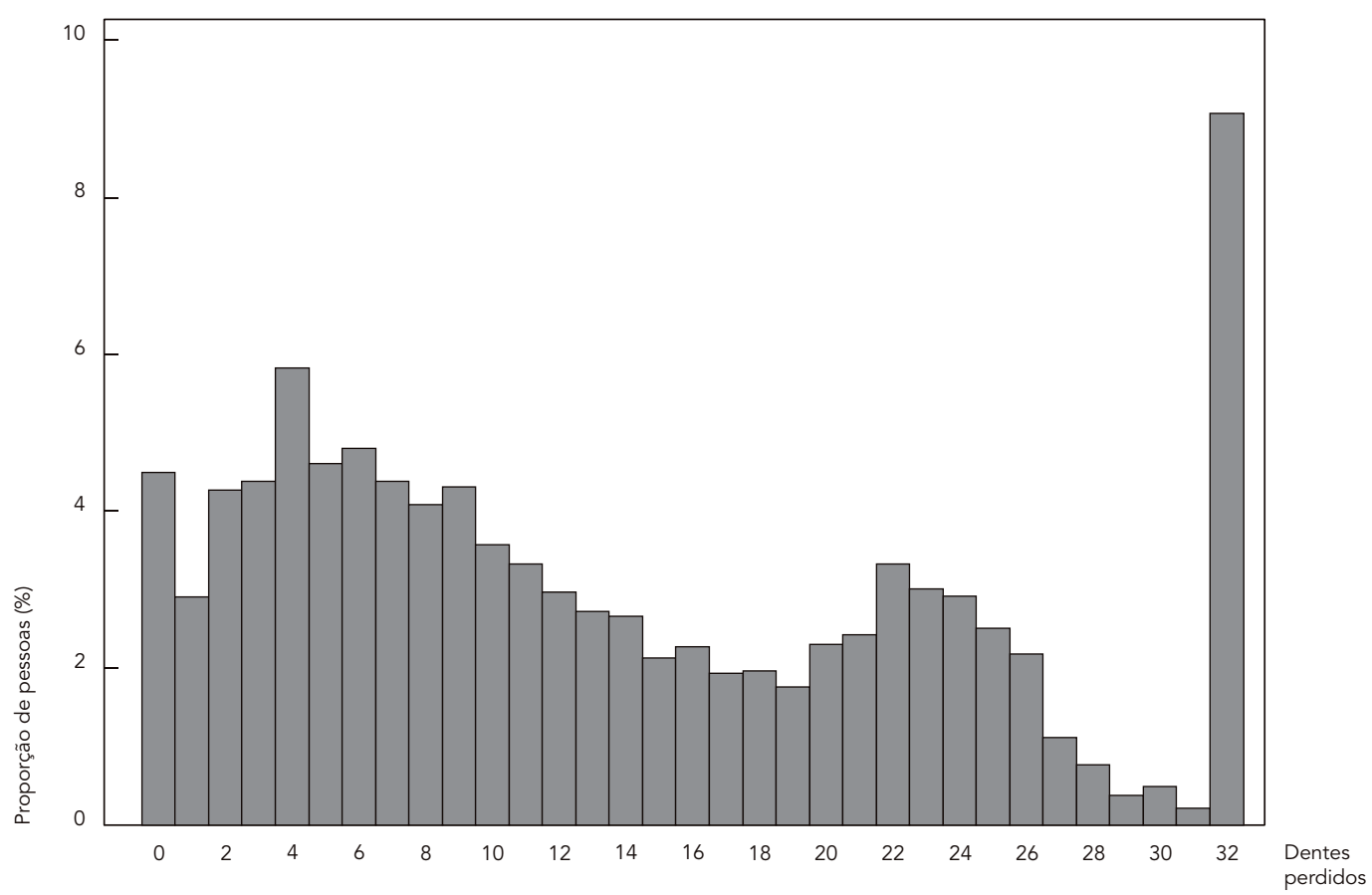

deu à moda da distribuição, resultado encontrado em 1.158 participantes do estudo. A média de dentes perdidos foi de 13,5 e a distribuição entre quartis foi de 5, 11 e 22 dentes para o primeiro, segundo e terceiro quartil, respectivamente.

Na Figura 3 são apresentadas as distribuições do número de dentes perdidos para cada uma das variáveis independentes e as respectivas medianas. Estas foram estatisticamente maiores para o gênero feminino, os moradores da zona rural, os de maior faixa etária, os pretos e pardos, os que apresentaram renda de até meio salário mínimo, os com escolaridade de até quatro anos, aqueles com três anos ou mais desde a última consulta odontológica e os que referiram ter utilizado serviços odontológicos da rede pública.

Na Tabela 1 são apresentadas as análises univariadas. Moradores de zona rural apresentaram uma prevalência de perdas dentárias (> 12 dentes) $15 \%$ maior que os moradores de regiões urbanas, enquanto os pretos e pardos apresentaram uma prevalência de perdas dentárias $14 \%$ maior que os brancos. As mulheres tiveram uma prevalência de perdas dentárias 1,4 vez maior que os homens. Foi clara e estatisticamente sig- nificante a existência de uma tendência de aumento da prevalência das perdas dentárias com a redução na escolaridade. Indivíduos com 5 a 8 anos de estudo e aqueles com até 4 anos de estudo apresentaram maiores prevalências de perdas dentárias quando comparados àqueles com escolaridade igual ou superior a 9 anos (razão de prevalência - RP de 1,54 e 1,84 respectivamente) Os mais pobres, os com consultas odontológicas realizadas há mais de dois anos e aqueles que se consultaram no serviço público tiveram uma maior prevalência de perdas dentárias (RP de $1,65,1,53$ e 1,28 respectivamente). Todas estas variáveis apresentaram forte associação com as perdas dentárias $(\mathrm{p}<0,01)$.

A Tabela 2 apresenta os modelos de análises multivariadas seguindo o modelo teórico de determinação (Figura 1). Perda dentária foi fortemente associada às condições sócio-econômicas e demográficas. Indivíduos residentes em zona rural, as mulheres, os mais pobres, aqueles com menor escolaridade e com idade mais avançada apresentaram prevalências maiores de perdas dentárias mesmo após o ajuste entre as variáveis. Apenas a diferença entre brancos 
Distribuição das perdas dentárias em adultos na faixa etária dos 35 aos 44 anos, de acordo com as categorias das variáveis demográficas, sócio-econômicas e de utilização de serviços odontológicos. Medianas e valores de p (testes de Mann-Whitney e Kruskall-Wallis)

3a. Localização

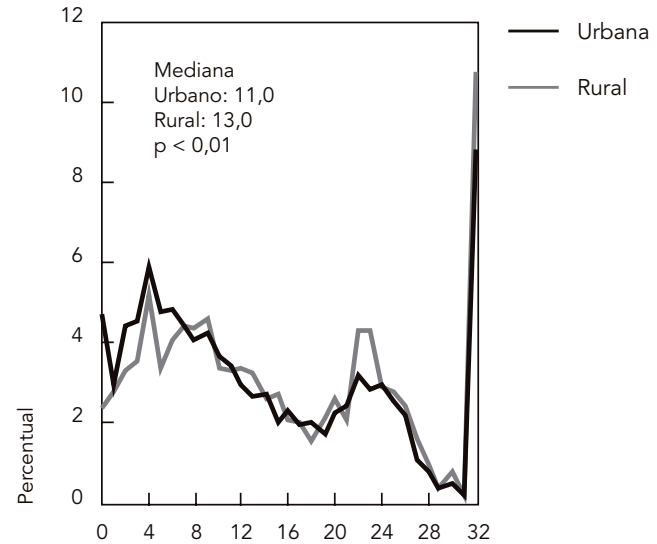

3c. Idade

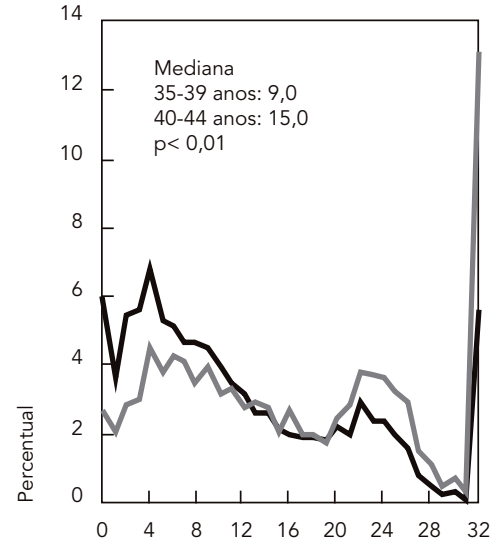

3b. Gênero

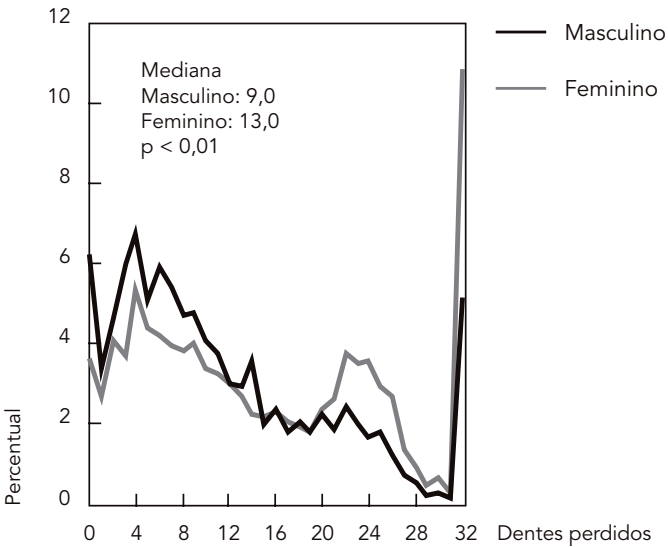

3d. Cor da pele

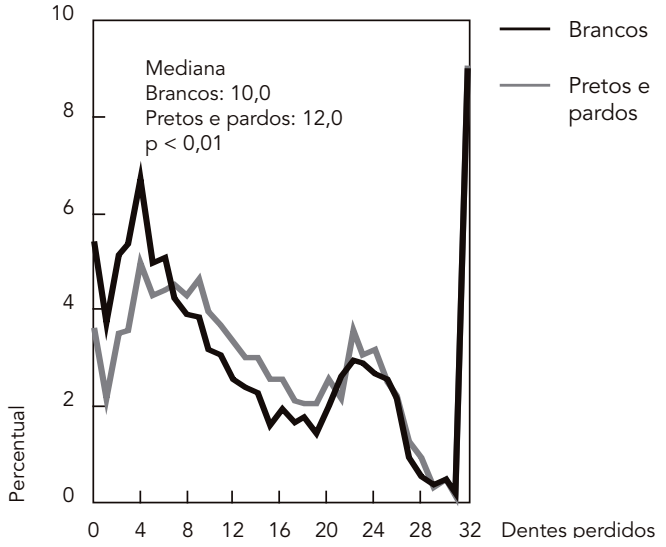

(continua)

e pretos e pardos perdeu a significância após o ajuste. Usuários do serviço público e aqueles que consultaram dentista há mais tempo também mantiveram maior prevalência de perda dentária após o ajuste por variáveis sócio-econômicas e demográficas.

\section{Discussão}

Este estudo revela alta prevalência de edentulismo $(9 \%)$ e elevado índice CPO-D médio $(20,44)$ na população brasileira entre 35 e 44 anos de idade. O número de dentes cariados, perdidos ou obturados identificado no estudo é maior que o encontrado no período de 1986 a 1989 na Inglaterra (19), Holanda $(17,4)$, Alemanha $(16,7)$, Austrália $(18,8)$ e Nova Zelândia (17) 20. 
3e. Renda per capita

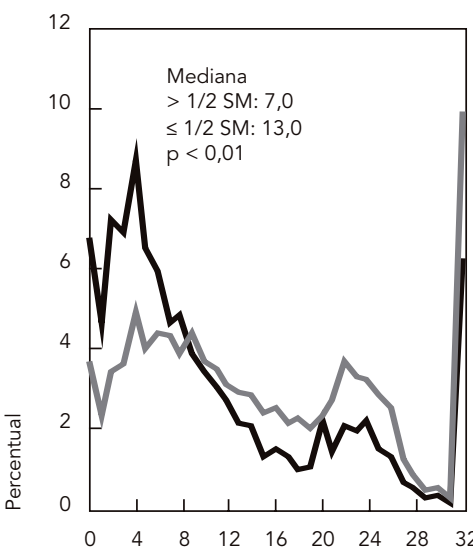

3g. Última consulta

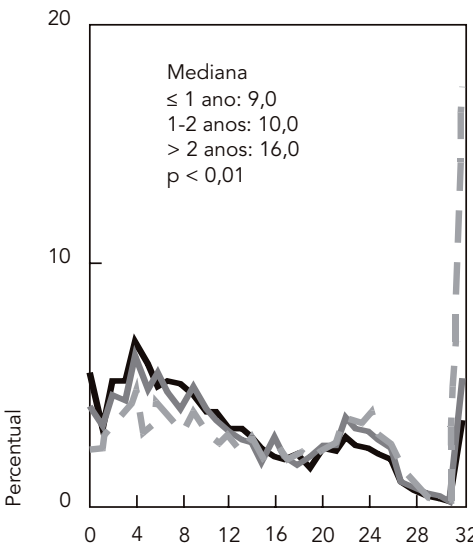

3f. Escolaridade

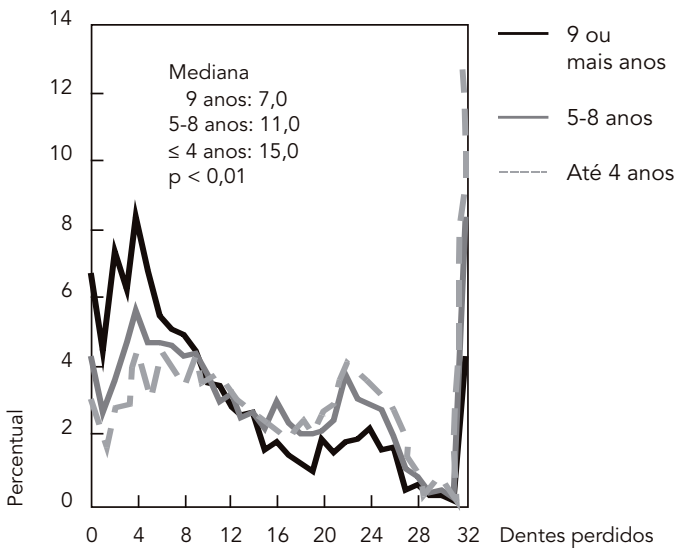

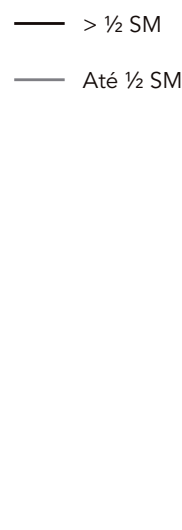

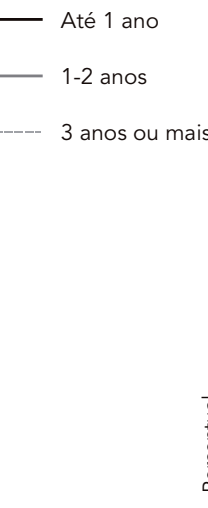

3h. Tipo de serviço

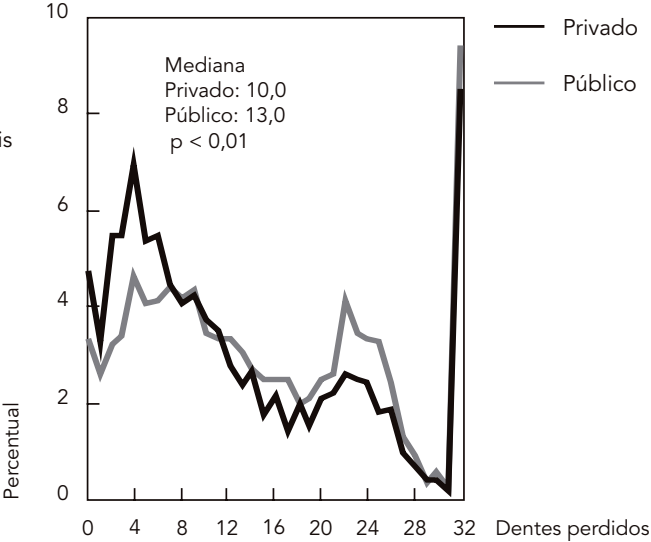

SM: salário mínimo

No período entre 2000 a 2003 o índice CPO-D médio de países como Tailândia $(6,13)$, Hong Kong $(7,14)$, Áustria $(14,7)$, Paquistão $(8,02)$ e Dinamarca $(16,7){ }^{21}$ para a mesma faixa etária também são bastante inferiores aos encontrados no Brasil. É importante salientar que na composição do índice de países como Áustria e Dinamarca, o componente "obturado" é o que apresenta a maior proporção, representando 83,7\% do índice na Áustria e 73,7\% na Dinamarca. Em Hong Kong e na Tailândia, o componente "P" tem a maior contribuição na composição do índice, respectivamente 54,6 e $57 \%$.
Numa comparação com valores internacionais também a proporção de edêntulos no Brasil é considerada elevada. No Líbano os edêntulos equivalem a $3,2 \%$ da população na faixa etária dos 35 aos 44 anos 22 e dados da OMS para os Estados Unidos da América 21 apontam que entre 1988 e 1991 cerca de $2,5 \%$ das pessoas com idade entre 35 e 39 anos eram edêntulas. Entre aqueles com 40 a 44 anos, o percentual chegava a 5,8\%.

A mediana do número de perdas dentárias encontrada neste estudo, equivalente a 11, contrasta com a proporção de perdas dentárias da população de 35 a 44 anos do Reino Unido, onde, 
Associação entre perdas dentárias e variáveis demográfica, sócio-econômica e de utilização de serviços odontológicos em adultos brasileiros.

\begin{tabular}{|c|c|c|c|c|c|}
\hline \multirow[t]{3}{*}{ Variável } & \multicolumn{2}{|c|}{ Dentes perdidos } & \multirow{3}{*}{$\begin{array}{l}\text { Total } \\
\text { n (\%) }\end{array}$} & \multirow[t]{3}{*}{ RP (IC95\%) } & \multirow[t]{3}{*}{$p$} \\
\hline & $\leq 12$ & $>12$ & & & \\
\hline & $n(\%)$ & n (\%) & & & \\
\hline \multicolumn{6}{|c|}{ Localização geográfica } \\
\hline Urbano & $6.174(54,7)$ & $5.111(45,3)$ & $11.285(88,1)$ & 1,00 & \\
\hline Rural & $729(48,0)$ & $789(52,0)$ & $1.518(11,9)$ & $1,15(1,04-1,26)$ & $<0,01$ \\
\hline \multicolumn{6}{|l|}{ Gênero } \\
\hline Masculino & $2.624(63,7)$ & $1.495(36,3)$ & $4.119(32,2)$ & 1,00 & \\
\hline Feminino & $4.283(49,3)$ & $4.409(50,7)$ & $8.692(67,8)$ & $1,40(1,33-1,47)$ & $<0,01$ \\
\hline \multicolumn{6}{|l|}{ Idade (anos) } \\
\hline $35-39$ & $4.332(62,2)$ & $2.630(37,8)$ & $6.962(54,3)$ & 1,00 & \\
\hline $40-44$ & $2.575(44,0)$ & $3.274(56,0)$ & $5.849(45,7)$ & $1,48(1,42-1,55)$ & $<0,01$ \\
\hline \multicolumn{6}{|l|}{ Cor da pele } \\
\hline Brancos & $3.374(57,3)$ & $2.519(42,7)$ & $5.893(46,0)$ & 1,00 & \\
\hline Pretos e pardos & $3.533(51,1)$ & $3.385(48,9)$ & $6.918(54,0)$ & $1,14(1,07-1,22)$ & $<0,01$ \\
\hline \multicolumn{6}{|c|}{ Renda per capita (salários mínimos) } \\
\hline$>1 / 2$ & $2.074(69,3)$ & $920(30,7)$ & $2.994(23,5)$ & 1,00 & \\
\hline$\leq 1 / 2$ & $4.782(49,2)$ & $4.944(50,8)$ & $9.726(76,5)$ & $1,65(1,53-1,79)$ & $<0,01$ \\
\hline \multicolumn{6}{|l|}{ Escolaridade (anos) } \\
\hline$>8$ & $2.431(69,7)$ & $1.056(30,3)$ & $3.487(27,4)$ & 1,00 & \\
\hline $5-8$ & $2.061(53,3)$ & $1.805(46,7)$ & $3.866(30,4)$ & $1,54(1,42-1,67)$ & $<0,01$ \\
\hline$\leq 4$ & $2.365(44,1)$ & $2.996(55,9)$ & $5.361(42,2)$ & $1,84(1,68-2,03)$ & $<0,01$ \\
\hline \multicolumn{6}{|c|}{ Tempo desde a última consulta (anos) } \\
\hline$\leq 1$ & $3.003(62,2)$ & $1.824(37,8)$ & $4.827(39,1)$ & 1,00 & \\
\hline $1-2$ & $1.641(56,9)$ & $1.245(43,1)$ & $2.886(23,3)$ & $1,14(1,08-1,21)$ & $<0,01$ \\
\hline$>2$ & $1.956(42,1)$ & $2.691(57,9)$ & $4.647(37,6)$ & $1,53(1,45-1,62)$ & $<0,01$ \\
\hline \multicolumn{6}{|l|}{ Onde se consultou } \\
\hline Privado & $3.402(59,5)$ & $2.314(40,5)$ & $5.716(48,2)$ & 1,00 & \\
\hline Público & $2.963(48,3)$ & $3.176(51,7)$ & $6.139(51,8)$ & $1,28(1,21-1,35)$ & $<0,01$ \\
\hline
\end{tabular}

em 1978, 65\% da população adulta apresentava 21 ou mais dentes presentes. Esse valor chegou a $86 \%$ em 1988 e a 93\% em 1998 23. Frazão et al. 14 estudando trabalhadores da educação no Estado de São Paulo, Brasil, para a mesma faixa etária, encontraram uma perda média de 11,57 dentes, enquanto Susin et al. 13 identificaram em adultos com 30 a 39 anos da região metropolitana de Porto Alegre, Rio Grade do Sul, Brasil, uma perda dentária média de 5,5 dentes e para a faixa de 40 a 49 anos, uma média de 10,6 dentes perdidos.

Apesar do importante declínio da cárie dentária e, conseqüentemente, de perdas dentárias dela decorrentes em crianças, registrado nas últimas duas décadas no Brasil, este fenômeno não é verificado entre os adultos. Isso é previsível, pois a população investigada neste estudo não foi exposta aos benefícios do flúor em todo o curso da vida. $\mathrm{O}$ abastecimento de água fluoretada e a disponibilidade de dentifrícios fluoretados apenas atingiram parcela expressiva da população brasileira a partir dos anos 1990 24, período em que a população investigada apresentava-se com idade entre 10 e 20 anos e, portanto, já com grande acúmulo de cárie e suas seqüelas. Mantendo-se, ou expandindo-se a fluoretação das águas e o consumo de dentifrícios fluoretados, espera-se um benéfico efeito de coorte para os adultos entre 35 a 44 anos nas décadas de 2010 e 2020 .

As mulheres apresentaram maiores índices de perdas dentárias. Dados relativos ao Líbano 22, Arábia Saudita 25 e Porto Alegre 13 corroboram nossos achados. Estudo longitudinal de base populacional realizado no Sul do Brasil revelou que os índices de cárie são maiores para as mulheres já a partir da adolescência 26 , o que pode explicar a diferença nas perdas na vida adulta. Outra hipótese explicativa desta diferença seria a maior utilização de serviços odontológicos por 
Associação entre perdas dentárias e variáveis demográficas, sócio-econômicas e de utilização de serviços odontológicos entre adultos brasileiros na faixa etária dos 35 aos 44 anos.

\begin{tabular}{|c|c|c|c|c|c|c|}
\hline \multirow[t]{2}{*}{ Variáveis } & \multicolumn{2}{|c|}{ Modelo 1} & \multicolumn{2}{|c|}{ Modelo 2} & \multicolumn{2}{|c|}{ Modelo 3} \\
\hline & $\operatorname{RP}($ IC95\%) * & $p$ ** & $\mathrm{RP}(\mathrm{IC} 95 \%)$ * & $p^{* \star}$ & $\mathrm{RP}(\mathrm{IC95 \% )}$ * & $p * *$ \\
\hline \multicolumn{7}{|l|}{ Bloco 1} \\
\hline Localização geográfica & & $<0,01$ & & & & \\
\hline Rural & $1,15(1,04-1,26)$ & & & & & \\
\hline \multicolumn{7}{|l|}{ Bloco 2} \\
\hline Gênero & & $<0,01$ & & $<0,01$ & & \\
\hline Feminino & $1,40(1,33-1,47)$ & & $1,39(1,33-1,46)$ & & & \\
\hline Cor da pele & & $<0,01$ & & 0,083 & & \\
\hline Pretos e pardos & $1,14(1,07-1,22)$ & & $1,05(0,99-1,11)$ & & & \\
\hline Idade (anos) & & $<0,01$ & & $<0,01$ & & \\
\hline $40-44$ & $1,48(1,42-1,55)$ & & $1,47(1,41-1,53)$ & & & \\
\hline Renda per capita (salários mínimos) & & $<0,01$ & & $<0,01$ & & \\
\hline Até $1 / 2$ & $1,65(1,53-1,79)$ & & $1,41(1,31-1,51)$ & & & \\
\hline Escolaridade (anos) & & $<0,01$ & & $<0,01$ & & \\
\hline $5-8$ & $1,54(1,42-1,67)$ & & $1,44(1,34-1,55)$ & & & \\
\hline$\leq 4$ & $1,84(1,68-2,03)$ & & $1,60(1,47-1,74)$ & & & \\
\hline \multicolumn{7}{|l|}{ Bloco 3} \\
\hline Tempo desde a última consulta (anos) & & $<0,01$ & & & & $<0,01$ \\
\hline $1-2$ & $1,14(1,08-1,21)$ & & & & $1,10(1,04-1,16)$ & \\
\hline$>2$ & $1,53(1,45-1,62)$ & & & & $1,33(1,26-1,39)$ & \\
\hline Tipo de serviço & & $<0,01$ & & & & $<0,01$ \\
\hline Público & $1,28(1,21-1,35)$ & & & & $1,09(1,05-1,15)$ & \\
\hline
\end{tabular}

Modelo 1: valores brutos; Modelo 2: variáveis do bloco 2 ajustadas entre si e pela variável do bloco 1; Modelo 3: variáveis do bloco 3 ajustadas entre si e pelas variáveis dos blocos 1 e 2 .

* Intervalo de confiança de $95 \%$ ajustado pelo desenho amostral;

** $\mathrm{p}$ valor $=$ teste de Wald

parte das mulheres, com conseqüente sobretratamento que resultaria na perda precoce do elemento dentário. Estudo realizado no Estado de São Paulo 14 identificou uma proporção mais elevada de perdas entre os homens, entretanto, esta investigação foi conduzida em um grupo específico de trabalhadores, não sendo um estudo de base populacional.

Quanto à localização geográfica, a mediana encontrada foi de 13 dentes perdidos no meio rural e 11 no meio urbano. Estudos realizados no Líbano 22 e em São Paulo 14 também apresentaram distribuição semelhante. Esta situação pode ser decorrente da infra-estrutura dos serviços oferecidos, onde para o meio rural normalmente se dispõe de menor oferta, menor acesso às tecnologias e à qualificação profissional, o que acaba por restringir os procedimentos odontológicos àqueles mutiladores. Não menos importante considerar que a distribuição de renda des- favorável aos indivíduos moradores no meio rural pode contribuir para estas perdas. Na Arábia Saudita 25 as perdas dentárias no meio urbano apresentaram a média de 4,29 e no meio rural de 3,57 dentes perdidos, porém a diferença não foi estatisticamente significativa. Os autores justificam a diferença encontrada devido à moderna infra-estrutura de serviços de saúde encontrada naquele país.

A maior proporção de mulheres nesta amostra pode ter levado à superestimação da prevalência de perdas dentárias para a população adulta brasileira. Segundo o IBGE, a distribuição por gênero na população brasileira nos anos 2002/2003 correspondia a aproximadamente $51 \%$ de mulheres e $49 \%$ de homens, enquanto neste estudo a proporção correspondeu a $67,8 \%$ de mulheres e $32,2 \%$ de homens. Uma das possibilidades do desequilíbrio entre os gêneros deveu-se provavelmente ao horário da coleta de dados, visto que 
durante o horário comercial aumenta a possibilidade de se encontrar mais mulheres que homens nas residências. Para minimizar essa distorção na amostra, no planejamento do estudo epidemiológico, os realizadores deveriam ter previsto essa possibilidade e definido estratégias para corrigila, tais como: incluir visitas no período noturno e em finais de semana.

Outra possível fragilidade do estudo pode estar vinculada ao padrão de vinte dentes presentes utilizado como referência. A proposta do arco dentário curto contempla a existência de vinte dentes presentes sem intervalos, correspondendo a um arco entre os segundos pré-molares, tanto superior quanto inferior. Neste estudo se considerou a presença de vinte dentes em ambos os arcos dentários, porém não foram considerados os elementos presentes, nem os possíveis intervalos entre os elementos.

As associações encontradas entre as perdas dentárias e os fatores sócio-econômicos considerados neste estudo corroboram os achados de Frazão et al. 14 para uma população do Estado de São Paulo e de Susin et al. 13 para uma população do Rio Grande do Sul.

Usuários de serviços públicos apresentaram maior prevalência de perdas dentárias, mesmo após o ajuste estatístico por outras possíveis variáveis confundidoras. Diante deste fato, duas hipóteses emergem: o quadro epidemiológico para esta faixa etária é grave, onde a carga de doença é muito grande e o serviço não está capacitado para absorver esta demanda, ou, o serviço público extrai mais dentes porque é um serviço com menor resolutividade, apresenta menores possibilidades de tratamento e a única alternativa para muitos casos é a extração. Convém ressaltar que somente a partir de 2003, com a implantação dos Centros de Especialidades Odontológicas, é que a endodontia e a prótese - especialidades determinantes no tratamento de dentes gravemente lesionados por cárie e na reabilitação dos danos severos aos elementos dentários ou na substituição nos casos de perdas - passaram a fazer parte das opções de tratamento oferecidas à população brasileira pelo serviço público. Estes resultados indicam a necessidade destes Centros de Especialidades Odontológicas oferecerem alternativas de próteses dentárias parciais, pois a oferta de próteses totais como única possibilidade de reparação protética pode estimular a execução de extrações dentárias.

Poder-se-ia supor que o serviço público, por atender uma população em teoria economicamente mais pobre e, conseqüentemente, com menor acesso ao atendimento odontológico, extrairia mais dentes. Porém, depois do ajuste na análise multivariada pelas variáveis sócio-econômicas, a prevalência de extrações no serviço público se reduziu de 28 para $9 \%$, percentual superior ao apresentado pela rede privada, o que indica ser ainda a extração uma característica de determinados tipos de serviços, em especial de municípios que apresentam piores indicadores sócio-econômicos 27.

Este estudo demonstra a necessidade de uma reorientação do serviço público odontológico, visando à prevenção de novas perdas dentárias na população adulta através de políticas públicas de saúde e prevenção voltadas especificamente para esta população. Existe, ainda, a real necessidade de reabilitar os danos já instalados da maneira mais equânime possível com a implementação de serviços de próteses. Também se tornam imperativas políticas de inclusão social, visto que os brasileiros têm na boca um retrato claro das desigualdades existentes no conjunto da sociedade brasileira.

\section{Resumo}

O objetivo deste estudo foi estimar a prevalência de perdas dentárias em adultos de 35 a 44 anos de idade. Além disso, foram testadas associações entre as perdas dentárias e condições demográficas, sócio-econômicas e com utilização de serviços odontológicos. Foram analisados os dados de 13.431 participantes do estudo epidemiológico nacional de saúde bucal realizado em 2002-2003. O número de perdas dentárias $(\leq 12$ e $>12)$ foi o desfecho investigado. As variáveis independentes incluíram localização geográfica, gênero, cor da pele, idade, renda per capita, escolaridade, tempo decorrido desde a última consulta odontológica e tipo de serviço utilizado. Foram estimadas as razões de prevalência bruta e ajustada através de regressão de Poisson. Edentulismo atingiu 9\% da amostra; a mediana de dentes perdidos foi igual a 11. Perdas dentárias foram fortemente associadas com indivíduos residentes em zona rural, com as mulheres, com os mais pobres, com os de menor escolaridade e com aqueles de idade mais avançada. Usuários do serviço público e aqueles que consultaram dentista há mais tempo também apresentaram maior prevalência do agravo mesmo após o ajuste por variáveis sócio-econômicas e demográficas.

Perda de Dente; Saúde Bucal; Desigualdades em Saúde; Adulto 


\section{Colaboradores}

P. R. Barbato, H. C. M. Nagano, F. N. Zanchet e A. F. Boing prepararam o banco de dados, realizaram as análises e redigiram o artigo. M. A. Peres idealizou o trabalho, realizou as análises e contribuiu com a redação do artigo.

\section{Referências}

1. Hung HC, Colditz G, Joshipura KJ. The association between tooth loss and the self-reported intake of selected CVD-related nutrients and foods among US women. Community Dent Oral Epidemiol 2005; 33:167-73.

2. Hebling E. Prevenção em odontogeriatria. In: Pereira AC, organizador. Odontologia em saúde coletiva: planejando ações e promovendo saúde. Porto Alegre: Editora Artmed; 2003. p. 426-37.

3. Dolan TA, Gilbert GH, Duncan RP, Foerster U. Risk indicators of edentulism, partial tooth loss and prosthetic status among black and white middleaged and older adults. Community Dent Oral Epidemiol 2001; 29:329-40.

4. Jovino-Silveira RC, Caldas Jr. AF, Souza EH, Gusmão ES. Primary reason for tooth extraction in a Brazilian adult population. Oral Health Prev Dent 2005; 3:151-7.

5. Cabral ED, Caldas Jr. AF, Cabral HA. Influence of the patient's race on the dentist's decision to extract or retain a decayed tooth. Community Dent Oral Epidemiol 2005; 33:461-6.

6. Burt BA, Eklund AS. Dentistry, dental practice and the community. 4th Ed. Philadelphia: W.B. Saunders Company; 1992.

7. Barros AJD, Bertoldi AD. Desigualdades na utilização e no acesso a serviços odontológicos: uma avaliação em nível nacional. Ciênc Saúde Coletiva 2002; 7:709-17.
8. Pinto VG. Saúde bucal coletiva. 4a Ed. São Paulo: Editora Santos; 2000

9. Lacerda JT, Simionato EM, Peres KG, Peres MA, Traebert J, Marcenes W. Dor de origem dental como motivo de consulta odontológica em uma população adulta. Rev Saúde Pública 2004; 38:453-8.

10. Klein H, Palmer CE. Dental caries in American Indian children. Washington DC: Government Printing; 1937. (Public Health Bulletin, 239).

11. Fundação de Serviços de Saúde Pública, Divisão Nacional de Saúde Bucal, Secretaria Nacional de Programas Especiais de Saúde, Ministério da Saúde. Levantamento epidemiológico em saúde bucal: Brasil, zona urbana, 1986. Brasília: Ministério da Saúde; 1988.

12. Departamento de Atenção Básica, Secretaria de Atenção à Saúde, Ministério da Saúde. Projeto SB Brasil 2003: condições de saúde bucal da população brasileira 2002-2003. Resultados principais. Brasília: Ministério da Saúde; 2004.

13. Susin C, Oppermann RV, Haugejorden O, Albandar JM. Tooth loss and associated risk indicators in an adult urban population from south Brazil. Acta Odontol Scand 2005; 63:85-93.

14. Frazão P, Antunes JLF, Narvai PC. Perda dentária precoce em adultos de 35 a 44 anos de idade. Estado de São Paulo, Brasil, 1998. Rev Bras Epidemiol 2003; 6:49-57. 
15. Departamento de Atenção Básica, Secretaria de Atenção à Saúde, Ministério da Saúde. Centro de especialidades: cidades atendidas. http://dtr2004. saude.gov.br/dab/saudebucal/cidades_atendidas. php (acessado em 10/Abr/2006).

16. World Health Organization. Oral health surveys: basic methods. $4^{\text {th }}$ Ed. Geneva: World Health Organization; 1997.

17. Käyser AF. Shortened dental arches and oral function. J Oral Rehabil 1981; 8:457-87.

18. Barros AJ, Hirakata VN. Alternatives for logistic regression in cross-sectional studies: an empirical comparison of models that directly estimate the prevalence ratio. BMC Med Res Methodol 2003; 3:21.

19. Kirkwood BR, Sterne JAC. Medical statistics. 2nd Ed. Massachusetts: Blacwell Science Ltd.; 2003.

20. Murray JJ. Comments on results reported at the second conference "changes in caries prevalence". Int Dent J 1994; 44:119-26.

21. World Health Organization. Global oral health. http:/ / www.whocollab.od.mah.se/countriesal phab.html (acessado em 13/Fev/2006).
22. Doughan B, Kassak K, Bourgeois DM. Oral health status and treatment needs of 35-44 year old adults in Lebanon. Int Dent J 2000; 50:395-9.

23. Downer MC. The improving dental health of United Kingdom adults and prospects for the future. Br Dent J 1991; 170:154-8.

24. Narvai PC, Frazão P, Roncalli AG, Antunes JLF. Cárie dentária no Brasil: declínio, polarização, iniqüidade e exclusão social. Rev Panam Salud Pública 2006; 19:385-93.

25. Al Shammery A, El Backly M, Guile EE. Permanent tooth loss among adults and children in Saudi Arabia. Community Dent Health 1998; 15:277-80.

26. Peres MA, Peres KG, Barros AJD, Victora CG. The relationship between family socioeconomic trajectories from childhood to adolescence and dental caries and associated oral behaviours. J Epidemiol Community Health 2007; 61:141-5.

27. Fernandes LS, Peres MA. Associação entre atenção básica em saúde bucal e indicadores socio-econômicos municipais. Rev Saúde Pública 2005; 39 : 930-6.

Recebido em 21/Set/2006

Versão final reapresentada em 01/Fev/2007

Aprovado em 09/Fev/2007 